\title{
ACC 270860
}

RECORDS ADMINISTRATION

HUMAN FACTORS IN INCIDENT INVESTIGATION

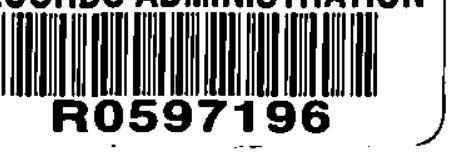

by

M. E. Armstrong

E. I. du Pont de Nemours and Company

Savannah River Laboratory

Aiken, SC 29808-0001

An abstract proposed for presentation and publication at the

Human Factors Society 33rd Annual Meeting

Denver, $\mathrm{CO}$

October 16-20, 1989

The information contained in this paper was developed during the course of work under Contract No. DE-AC09-76SR00001 with the U. S. Department of Energy. By acceptance of this paper, the publisher and/or recipient acknowledges the U. S. Government's right to retain a nonexclusive, royalty-free license in and to any copyright covering this paper, along with the right to reproduce and to authorize others to reproduce all or part of the copyrighted paper. 


\author{
Mark E. Armstrong \\ E. I. du Pont de Nemours \& Co., Inc. \\ Savannah River Laboratory \\ Aiken, SC 29808
}

\begin{abstract}
The Savannah River Plant (SRP), located in South Carolina, is a key Department of Energy production and research facility for nuclear materials. Incident investigations performed at the Savannah River Plant showed the cause of approximately $75 \%$ of all operating incidents to be human error. The technical incident reporting system in place required the investigator to list the cause of an incident in broad terms (i.e., Personnel Error, Equipment Error) and to categorize it according to subclassifications (i.e., Operator Error, Supervisor Error, Mechanic Error). The reporting system, using these classifications, told "what happened" during an incident and "who was involved", instead of telling "why" an incident occurred. The high rate of human error as the cause of incidents indicated that further analysis was in order.

Human factors personnel in the Facility Safety Evaluation Section (FSES - an oversight organization with emphasis on non-reactor facilities) wanted to determine the causes of human error a way that would identify why the errors occurred. To satisfy these needs FSES developed a root cause analysis method of incident investigation for SRP. Root cause analysis consists of two parts; the first being Events and Causal Factor (E\&CF) Charting; and the second, Root Cause Coding using a Root Cause Tree. The objectives were to provide a systematic method for identifying the root causes of a given incident in order to make detailed recommendations to prevent its recurrence, and to provide a database of incident root causes for identifying problem areas across incidents. Root cause analysis would allow the incident investigator to state "why" an incident occurred using detailed cause codes (i.e., Incomplete Training, Labels Less Than Adequate). Root cause trending would enable FSES to track the causes of human error, recommend solutions, and track corrective actions. FSES developed a one day workshop to train several hundred incident investigators at SRP how to perform investigations using the root cause analysis method. This presentation will discuss the development and implementation of a root cause analysis system at SRP by FSES human factors professionals.
\end{abstract}




\title{
HUMAN FACTORS IN INCIDENT INVESTIGATION
}

\author{
Mark E. Armstrong \\ E. I. du Pont de Nemours \& Co., Inc. \\ Savannah River Laboratory \\ Aiken, SC 29808 \\ PRESENTATION SUMMARY
}

\section{Objectives of Work}

The Savannah River Plant (SRP), located in South Carolina, is a key Department of Energy production and research facility for nuclear materials. The plant produces materials used in nuclear weapons, naval ships, space satellites, and medicine. Covering over 300 square miles, SRP facilities consist of reactor fuel and target fabrication facilities; nuclear materials production reactors; separations facilities for processing irradiated nuclear material; waste storage and processing facilities; the Savannah River Laboratory, a research and development laboratory; administrative facilities; and other support facilities required for plant operation.

The Savannah River Plant operations facilities have established systems for investigating and reporting incidents that occur. At SRP, an incident is defined as any deviation from normal operation. The technical incident reporting system previously in place required the investigators to determine and list the cause of an incident in broad terms (i.e., Personnel Error, Equipment Error), and categorize the cause according to subclassifications (i.e., Operator Error, Supervisor Error, or Mechanic Error). The reporting system, using these classifications, told "what happened" during an incident and "who was involved", instead of telling "why" an incident occurred. The investigators did not receive formal training on how to carry out an investigation. As a result, recommendations from the investigations were often too general (e.g., Remind all personnel of the importance of following procedures). These types of recommendations were easy to implement, but were not sufficient to prevent incident recurrence.

Incident investigations at SRP showed the cause of approximately $75 \%$ of all operating incidents to be human error in the non-reactor facilities. The high rate of human error as the primary cause of incidents indicated that further analysis of the human error was in order. Human factors personnel in the Facility Safety Evaluation Section (FSES - an oversight organization with emphasis on non-reactor facilities) wanted a way to determine the causes of human error that would identify why the errors occurred. To satisfy these needs FSES developed a root cause analysis method of incident investigation for SRP non-reactor facilities. The objectives were to provide a systematic method for identifying the root causes of incidents in order to make more explicit recommendations to prevent their recurrence, and provide a database of incident root causes for identifying problems. Root cause analysis would allow the incident investigator to state "why" an incident occurred using detailed cause codes (i.e., Incomplete Training, Labels Less Than Adequate). Root cause trending would enable FSES to track the causes of human error, recommend solutions, and track corrective actions.

\section{Objectives of the Presentation}

The objectives of the presentation are to provide background information about the Savannah River Plant; provide information concerning the old system of performing incident 
investigation; explain the reasons for developing the root cause method of incident investigation; explain why human factors personnel from a safety evaluation group were heavily involved in development; explain the root cause method in general; provide information about the implementation of the system; and explain the results and conclusions drawn from the work.

\section{Method and Approach}

The root cause analysis system at Savannah River was developed by FSES human factors professionals, based on initial work by members of the Reactor Safety Evaluation Section (RSES) (Paradies \& Busch, 1986). Elements of several incident investigation systems used in the nuclear industry were incorporated. FSES developed the Root Cause Tree, a Root Cause Analysis Handbook, and a Root Cause Analysis Workshop based on the needs of the investigators and human factors principles.

Root cause analysis consists of two parts; the first being Events and Causal Factor (E\&CF) Charting; and the second, Root Cause Coding using a Root Cause Tree. E\&CF Charting provides the incident investigator with a method for organizing and analyzing information gathered during an investigation. Gaps in knowledge or sequence of events can be readily identified using EC\&F charting. Root cause coding involves categorizing the causal factors of an incident using the Root Cause Tree. The Root Cause Tree is a decision tree that contains the root cause codes.

Implementation of root cause analysis into the incident investigation system at SRP is being coordinated by FSES personnel. Once the root cause analysis method was developed, FSES tested the method by reinvestigating a number of incidents that had been done according to the previous system. The root cause method provided greater detail as to why the incidents occurred and allowed more detailed recommendations to be made to prevent recurrence. FSES developed a one day workshop to train several hundred incident investigators at SRP to perform investigations using the root cause analysis method. The workshop centers around performing a simulated root cause investigation of an actual incident that occurred at SRP.

\section{Results and Conclusions}

Root cause analysis is still in the implementation process at SRP. Initial feedback on incident investigations using the new system has been positive. FSES will maintain a root cause database for the non-reactor facilities at SRP. The database will allow the analysis and trending of incident causes by FSES. From this information, routine reports will be made to management regarding safety performance. FSES human factors personnel will use the human performance data to identify specific areas for further evaluation and improvement, such as procedures or interface design. FSES will make recommendations for reducing human error in the specified areas.

\section{Possible Applications}

The root cause analysis system at SRP will provide real world data on the types of specific system problems that cause human error. The FSES root cause system could be tailored to applications at other sites and in other industries as an incident investigation tool, system analysis tool, and a source of human error data. 